Research Article

\title{
Composition and Optimization of Higher Education Management System Based on Data Mining Technology
}

\author{
Jingnan $\mathrm{Hu}$ (D) $^{1}$ and $\mathrm{Hui} \mathrm{Li}^{2}$ \\ ${ }^{1}$ School of Accounting, Hainan Vocational University of Science and Technology, Haikou, Hainan 571126, China \\ ${ }^{2}$ Zhongjie Zhonghuan Certified Public Accountants (Special General Partnership) Hainan Branch Haikou, \\ Hainan 570203, China \\ Correspondence should be addressed to Jingnan Hu; hjn010@njau.edu.cn
}

Received 5 September 2021; Revised 23 October 2021; Accepted 25 October 2021; Published 8 November 2021

Academic Editor: Rahman Ali

Copyright $\odot 2021$ Jingnan $\mathrm{Hu}$ and Hui Li. This is an open access article distributed under the Creative Commons Attribution License, which permits unrestricted use, distribution, and reproduction in any medium, provided the original work is properly cited.

\begin{abstract}
In order to improve the low efficiency of higher education management and the unequal distribution of curriculum resources, facing the actual situation of higher education management, a higher education management system based on data mining technology is constructed and optimized. Using the correlation between MVC components of higher education management platform and the support of data mining technology, this paper constructs the MVC model 2 framework of higher education management platform which is a powerful framework used for developing large-scale projects with ease. We collect the data structure in the database, extract the evaluation indicators of higher education management system, separate the display results and business logic, and improve code reusability. We adopt data input, curriculum management, curriculum scheduling, teacher scheduling, and system maintenance and help to form and optimize the higher education management system. The experimental results show that the constructed and optimized higher education management system can maintain good scheduling performance and provide better teaching services for teachers and students.
\end{abstract}

\section{Introduction}

The central objective of higher education is to guide students to establish a correct outlook on life, values, and world, to arm themselves with scientific ideas and knowledge, and to enable students to become creative talents with both ability and political integrity [1]. Traditional education and teaching adopt manual course management and teach through temporary course transfer of teachers. Timetable arrangement is the result of efficient integration of teacher resources, classroom resources, and time resources and cannot produce any conflict [2]. Therefore, it is of great significance to construct and optimize the higher education management system according to the existing higher education management information, and to adjust the curriculum arrangement properly.

In this paper, we propose the composition and optimization of higher education management system based on data mining technology. We build a higher education management platform MVC (Model View Controller) framework model, which is a very mainstream form of design at this stage. The MVC framework was introduced by Sun Corp. Earlier, a J2EE-standard design model was widely used in many web applications. With the continuous expansion of the field of use, the product has been recognized and loved by more and more developers. Firstly, the overall scheduling mode of the platform is optimized based on the principle of priority to improve the efficiency of intelligent scheduling of information, and the MVC framework of the higher education management platform is used to achieve high-quality human-computer interaction goals. The proposed system consists of various modules that include data input, curriculum management, curriculum scheduling, teacher scheduling, and system maintenance and helps to form and optimize the higher education management system. 


\section{Related Work}

In the recent years, many researchers have focused on the area of education management using data mining and machine learning technologies. In this section, we are going to discuss some of the existing works in the area. Gutsu et al. [3] put forward the subjective attitude of college teachers towards the reform of higher education. At the beginning of the $21^{\text {st }}$ century, Russia's higher education system carried out a large-scale reform. The implementation of the concept of modernization has been entrusted to university teachers, and the actual results of the announced changes depend to a large extent on how teachers view and treat the ongoing changes. The subjective attitudes of teachers in higher education were considered along with other factors such as introduction of competency-based approaches in higher education, the reduction of classroom time and work, the technicalization of the learning process, and the increase in the proportion of distance training, including full-time students. According to the subjective opinions of the teachers involved in the study, most of the changes have no significant impact on the actual situation of higher education. The empirical data obtained are of great significance to psychology, management, and higher education management. Djuraevna [4] put forward the formation of student management activities under the higher education management system. The paper introduces the concept of education management, the scientific work of scientists on management issues, the principles of modern management, the organization of experimental work in the research process, and the results of implementation in practice. Chen [5] focused on optimization of clinical nursing management system. They proposed to combine data mining technology with nursing information for optimizing the nursing information system. They used the hospital's existing care system and improved the Bayesian algorithm and data processing to improve various components and their functionalities including information systems related to patients, medical orders, execution, expenses, physical sign, and more. Wang [6] investigated the use of big data and big data analytics in education management. They concluded that, due to the immature technology, there are technical defects in the management systems in the education sector and proposed an optimization scheme which includes data normalization method, optimizing the data clustering, enhancing the prediction accuracy, and simplifying calculations and computations. Yin [7] applied data mining technology to students' information management system to improve the students' evaluation data and evaluation modules and point out the factors that have a negative impact on students' development. They propose an improved genetic fuzzy clustering algorithm and apply it to the students' information management system to make the students' evaluation and decision making better. Similarly, Aman et al. [8] applied data mining using decision tree algorithms to the problem of predicting students' academic performance on the basis of their educational record to know whether the student will drop out or will successfully complete the academic degree program.
In literature $[9,10]$, different categories of online and offline learning and educational systems are available, such as Sakai, Moodle, Coursera, Google Classroom, and many others which have different features with respect to online lecture session and duration, recording, number of participants, class scheduling, and coordinating software.

\section{Treatment of Higher Education Management System under Data Mining Technology}

3.1. Construction of Higher Education Management Platform under Data Mining Technology. Figure 1 represents the interrelationships between MVC components of the higher education management platform. In the framework of MVC $[11,12]$, the key task of the client is that the user sends the corresponding request to the back-end server through operation of the client. When transporting a request, the HTTP protocol is used to transmit the request to the server. After receiving the request, the server processes the request according to the content of the request and returns the processing results to the client by using various modes. The user can receive the background feedback results in the interface. In this process, the backstage database layer is the data layer [13], which is responsible for the communication and interaction between the database and the user or administrator.

The view layer can feed back the data transmitted in the backstage server in several forms to the platform interface, and the user can easily view and read the data. Higher education management platform client can be shown as a combination of multiple small procedures. The model layer is a key layer in the design form of MVC framework for higher education management platform $[14,15]$. On the premise of receiving the corresponding request of the view layer, the corresponding business logic analysis shall be implemented for the program according to the business logic of the program. In general, the corresponding database basic operation shall be adopted between the model layer and the database, and the relevant data structures within the database operation shall be collected so as to separate the display results from the business logic. The key task of the control layer is to fuse the user's requests and access of the two layers so that the corresponding business logic results can be processed quickly.

To correct the application state of the higher education management platform on the web, we improve the standard MVC pattern, which is MVC Model 2, as shown in Figure 2.

The information intelligent scheduling platform of higher education management system designed in this paper uses Dreamweaver and other tools to develop the static part of the web page, uses flash to develop the software of animation part, uses Java and Jsp to complete the platform interaction, and adopts the open source software architecture Struts, Spring, Hibernate.

The hierarchical structure of the platform is divided into presentation layer, business logic layer, data persistence layer, and domain model layer, and each layer has its own responsibilities and tasks. The presentation layer is the closest layer structure to the user, which takes on the 

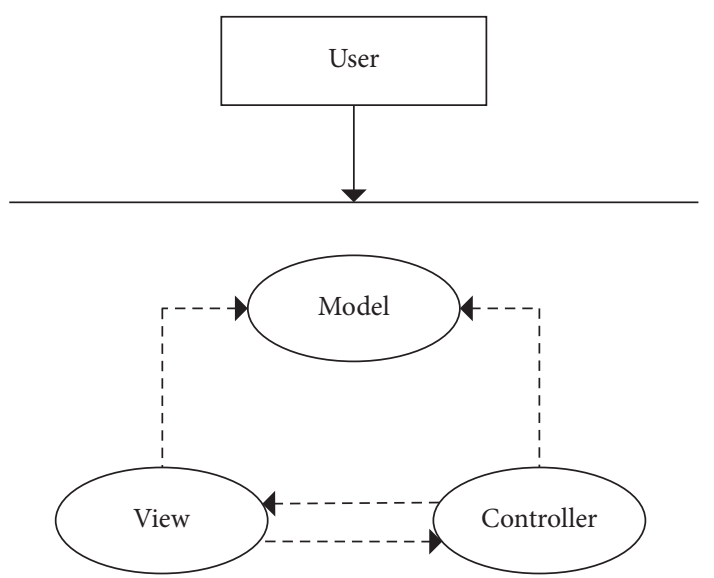

FIGURE 1: Correlation between the MVC components.

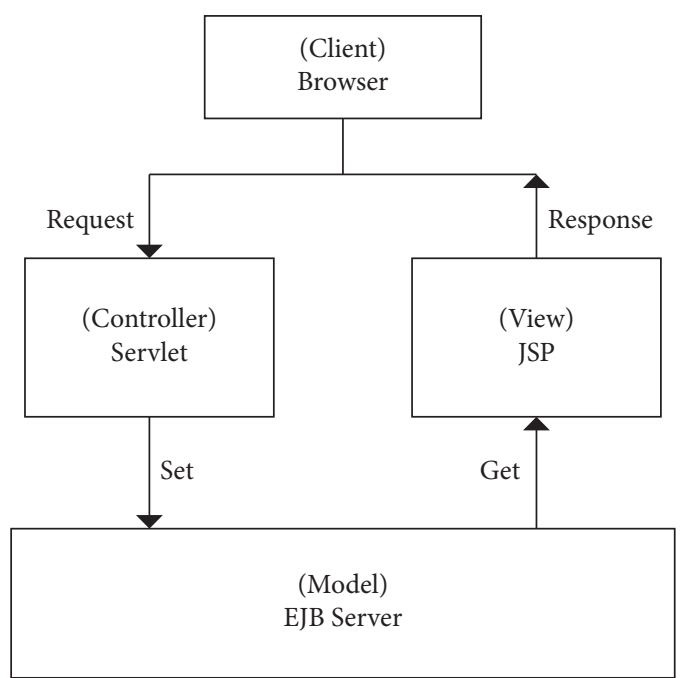

FIgURE 2: Higher education management platform MVC model 2 framework.

function of displaying the interactive interface for the user, manages all kinds of requests submitted by the user, and gives corresponding feedback.

The business logic layer has the characteristics of processing platform program business logic, business calibration, and transaction management. It clarifies the environment from presentation layer to business logic layer.

The data persistence layer encapsulates the data orientation details, provides effective services to the business logic layer, and supplies the continuous object-relational mapping to Java, which maps the objects in each module of the platform to the relational database entity to improve the comprehensive use efficiency of the platform.

Domain model layer can get the collection of business goals in business domain through platform analysis, and it can move between different levels. It can exchange information on each level of MVC framework.

The information intelligent dispatching platform of higher education management system fully considers the data interface with other teaching information management platforms. All data are accessed from the existing teaching plan management system and course scheduling system to prevent repeated data input. At the same time, as a further expansion of the higher education management course scheduling system, the intelligent scheduling platform fills the defects of the course scheduling system in adjusting the timetable. The data exchange process between the intelligent scheduling platform and relevant subsystems is shown in Figure 3.

Some schools have not constructed the complete higher education management information dispatch platform and have not had the existing data access. In the process of operation and management information scheduling platform, the workload of general data input is very large, and errors are very easy to occur. For example, for a 6000person-scale school, input a semester full of higher education management information, to keystroke more than 150,000 times. When designing the input board, the platform will be close to the manual operation, conform to the daily work rules of the Academic Affairs Office, and use the input coding strategy to minimize the input workload, reduce the error rate, and effectively play the advantages of computer management, for example, the input of class information and curriculum information, such as input coding and network independent search. Find mode is also very rich and easy to operate.

According to the daily work of higher education management, the data input module, curriculum schedule management module, curriculum scheduling module, classroom scheduling module, system maintenance, and help module are established in the intelligent scheduling platform of higher education management information, as shown in Figure 4.

The data input module represents the interface which will be used by the administrators and teachers etc. to enter data or access data and information regarding any of the other modules and features of the information system. Curriculum management is the overall control of the curriculum of higher education, with the most basic curriculum elements: curriculum progress, teacher's name, classroom, time, and class. In the dispatching platform, it can quickly find the required curriculum information, browse, revise, print, and take charge of receiving the data of the course scheduling system. The data of a student's progress and other reports is managed by this module and can make the teachers of higher education lesson operate the curriculum as a whole and help them to better prepare the curriculum.

Classroom scheduling board can arrange tasks by borrowing teachers and examination room. Time and number of seats will be input into the classroom. If the user specifies to borrow a classroom, the platform will print out the conflict status of the classroom, allowing the user to complete the classroom scheduling task. The classroom scheduling process is shown in Figure 5.

Curriculum scheduling plan refers to the management of courses and classes. If you want to transfer courses to higher education, you can use the mouse to drag it to a new position, and the platform can evaluate whether there is a conflict or not. To force the transfer of courses, the remaining courses will be transferred, and the platform will 


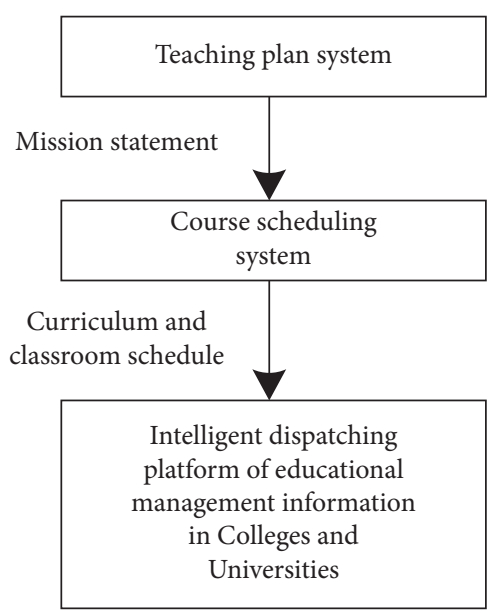

FIgURE 3: Data exchange process for intelligent scheduling platforms and systems.

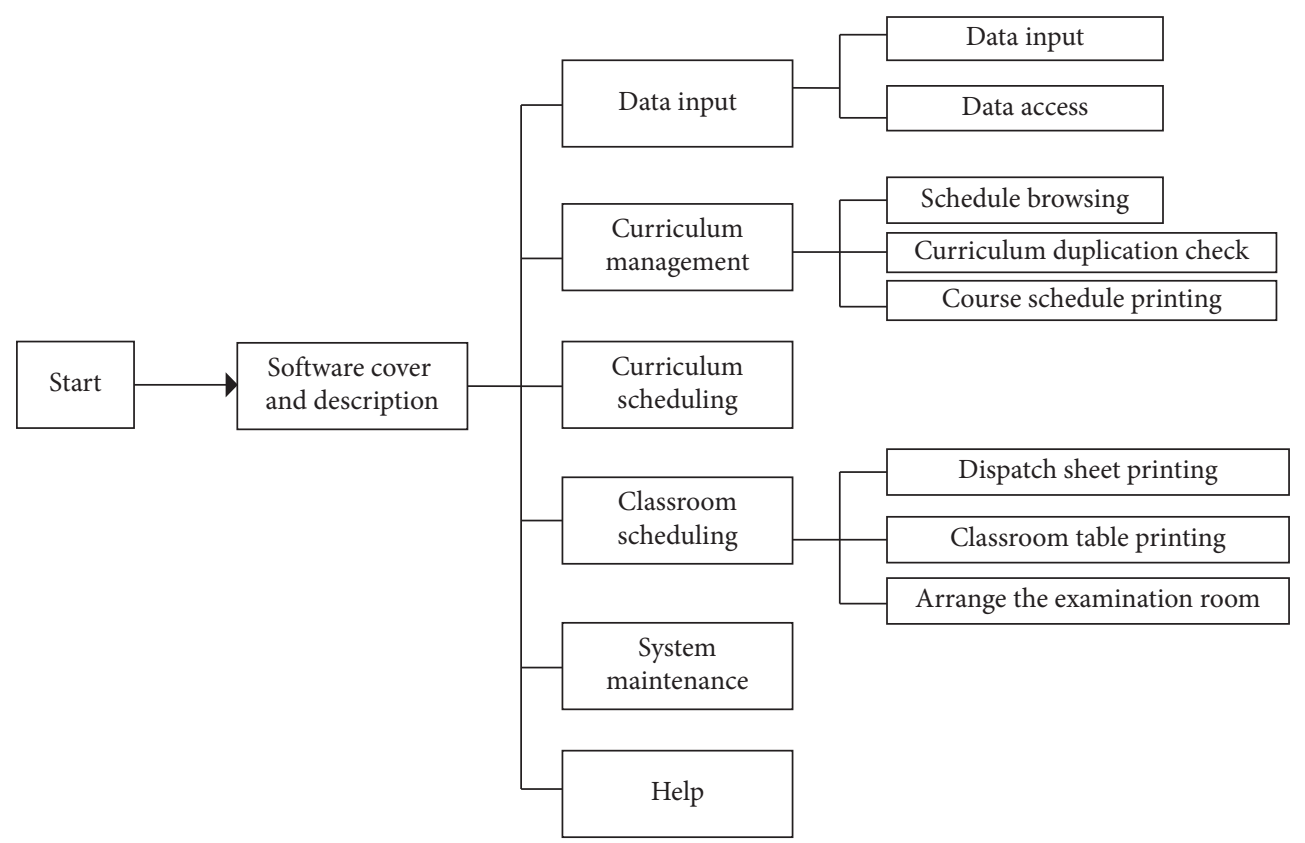

FIGURE 4: Structure of intelligent scheduling platform.

print conflict information to facilitate the implementation of followup course progress. When higher education classrooms are occupied, the platform will intelligently schedule the available classrooms for teachers to choose from. The course scheduling process is shown in Figure 6.

The system maintenance module is an optional module that could be developed to make the common maintenance related tasks easier for the administrators who are not skillful enough to understand the underlying architecture of the system. This may include the interface to change configurations and other tasks like adding new tables to the database.

3.2. Features Extraction. As a typical deep learning algorithm model, data mining technology has been widely used $[16,17]$. At present, it is widely used in various research fields. Its weight sharing and local connection characteristics can effectively reduce the number of parameters and computational complexity. Data mining technology includes multiple hidden layers, which can convert the input multidimensional image into other vector data, mainly composed of convolution layer and sampling layer.

Since the structure of data mining technology is locally connected and the weights are shared, compared with BP neural network [18], the weights of data mining technology are more complex. In the following, the weights are updated by accelerating the adjacent gradient algorithm to comprehensively improve the convergence speed.

The objective function of data mining technology is

$$
w^{k+1}=P_{\text {prox }} \times f\left(w^{k}\right) .
$$




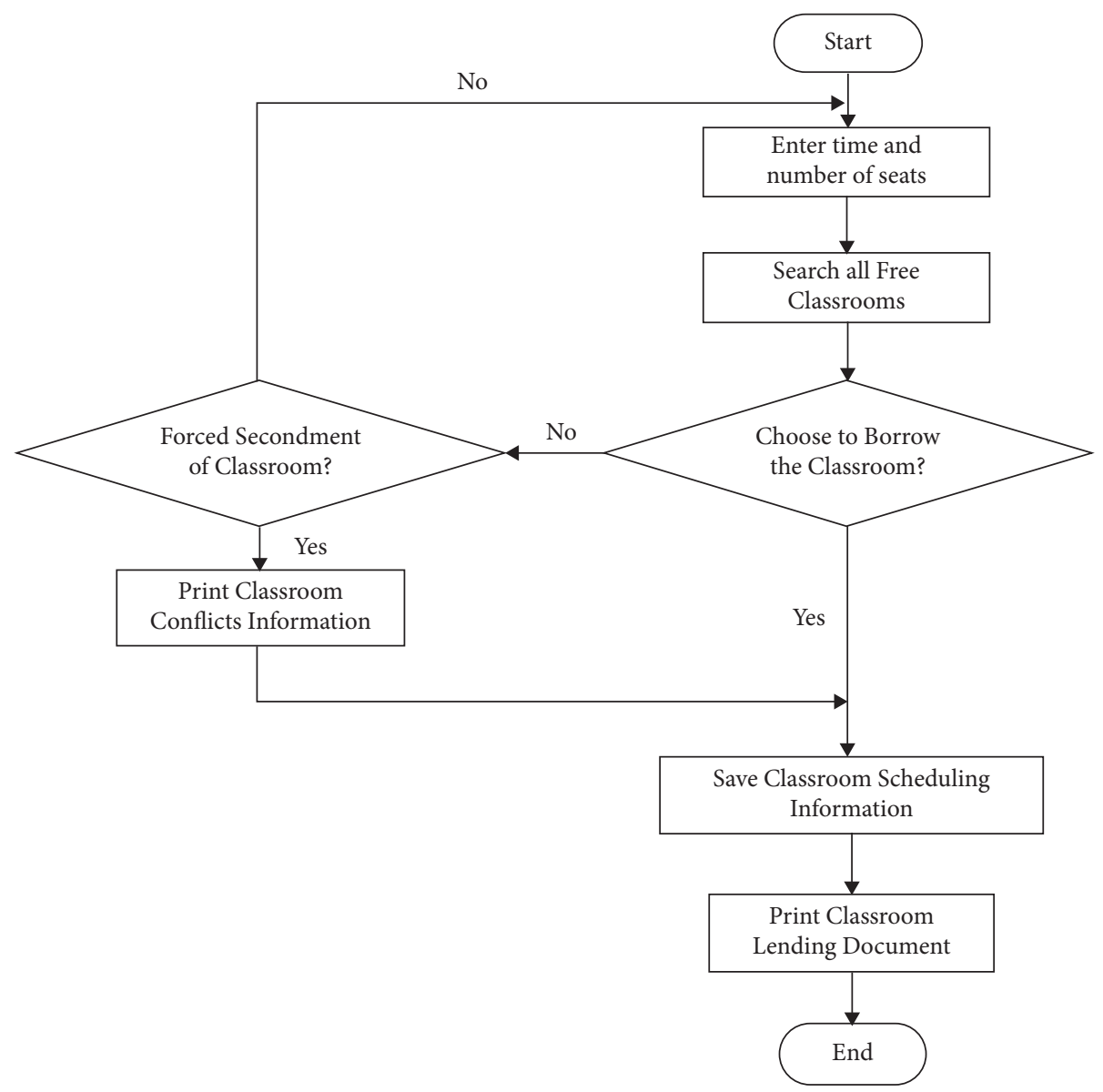

FIGURE 5: Classroom scheduling process diagram.

In the formula, $P_{\text {prox }}$ represents the number of iterations and $f\left(w^{k}\right)$ represents the network weight. Calculate the gradient value $\delta^{\text {out }}$ of the output node of the final output layer. The specific calculation formula is as follows:

$$
\delta^{\text {out }}=z^{\text {out }} \times * f^{\prime}\left(z^{\text {out }}\right) \times w^{k+1} .
$$

In the formula, $z^{\text {out }}$ represents the input value size of the output node of data mining technology and $f^{\prime}\left(z^{\text {out }}\right)$ represents the derivative of the activation function $f$. In general, the activation function uses the sigmoid form and is deduced based on it, and then the gradient of the hidden layer can be obtained [19]. Through the obtained gradient, the weight parameters are continuously updated during the training process, so as to promote the network learning to obtain the characteristics with certain value. Among them, the gradient of the previous layer can be deduced as

$$
\delta^{\text {out-1 }}=z^{\text {out-1 }} \times|t-y|^{2} \text {. }
$$

The actual output value $y$ of the network can be defined as $y=f\left(z^{\text {out-1}}\right)$, so formula (3) can be expressed in the following form:

$$
\delta^{\text {out-1 }}=z^{\text {out-1}} \times\left|t-f\left(z^{\text {out }}\right)\right|^{2}
$$

In order to better realize the feature extraction of evaluation indicators, it is necessary to spread the data mining technology, add an encoder to the input vector, and use the nonlinear mapping function to obtain the coding related to the input, that is, the feature expression of the input data. In order to make the expression more vivid, the input vector is approximated by the output of the decoder.

Since the features formed by the encoder are applied to the input data of the next hidden layer, the hidden layer will accelerate the training of adjacent algorithms. When training the existing hidden layer, the trained parameters do not need to be changed. The number of layers of the network and the number of nodes in the hidden layer need to be adjusted according to the actual situation. After pretraining, the global network is adjusted by accelerating the proximity algorithm. At the same time, a decoder needs to be added at the last layer of the network to make the expected output closer to the input vector. The whole network is mainly trained by accelerating proximity algorithm. The computational complexity of the algorithm is an important standard to measure the performance of the algorithm. The whole algorithm mainly includes the inversion of the matrix, and the computational complexity can be expressed as $o\left(n^{3}\right)$. $n$ represents the dimension of feature vector, so most of the time it is spent in the composition of moment inversion and data size transformation in the process of training. 


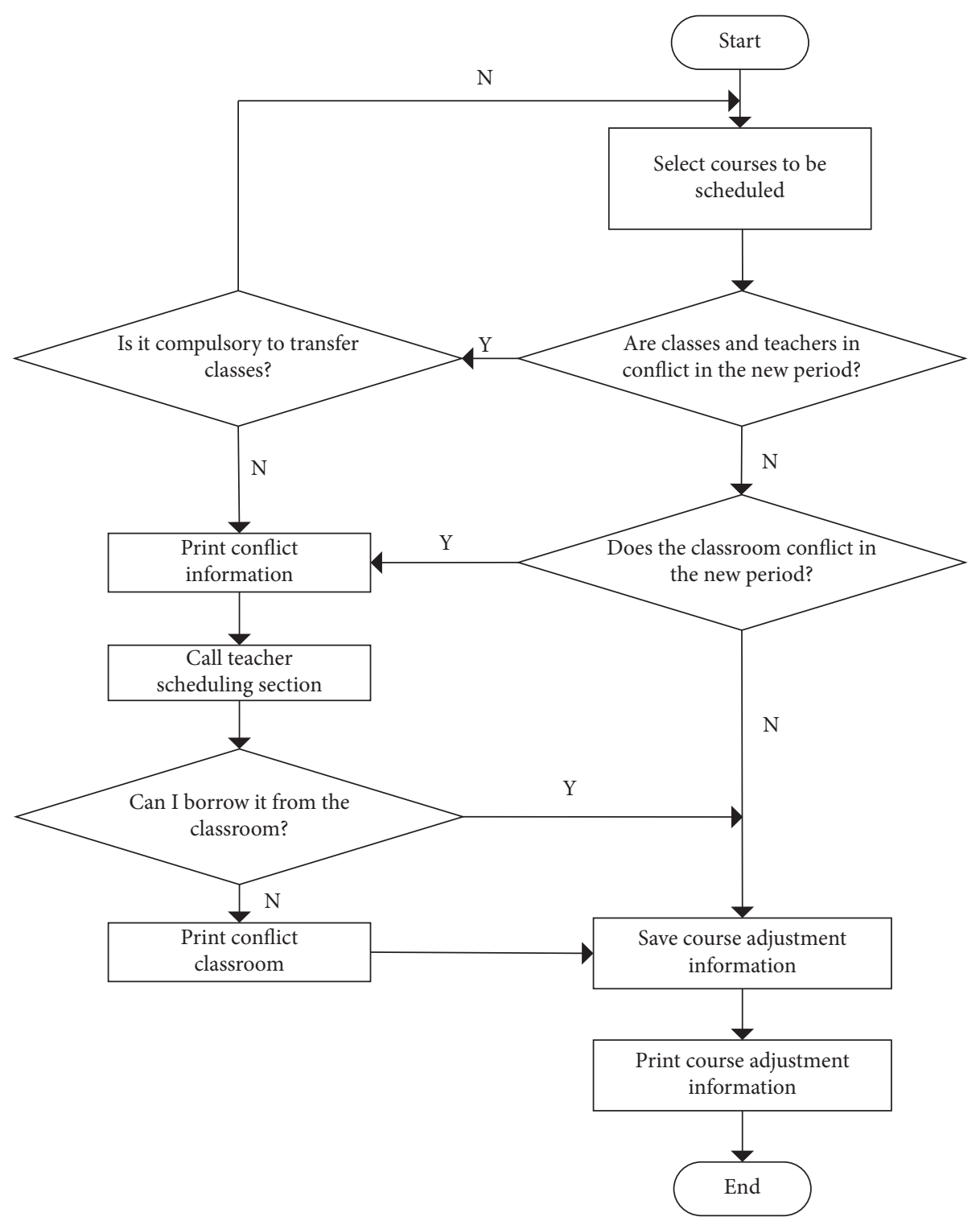

FIGURE 6: Higher education management scheduling process.

The feature dimension of the evaluation index extracted by data mining technology can be expressed as $x_{1}, x_{2}, \ldots, x_{i}$, where $x_{i}$ represents the number of $i$ element values in the vector center and $i=1,2, \ldots, n$ represents the input dimension of the network. The increase of input dimension will lead to the exponential increase of the extracted feature vector dimension of higher education management system evaluation index. When extracting features through data mining technology, the dimension of feature vector is mainly established by the total number of network output nodes of higher education management system [20].

When the data mining technology is combined with the least squares iterative algorithm, the objective function is convex optimization, which is solved by the accelerated neighbor algorithm. At the same time, the extracted indexes are input into the multilayer perceptron for classification to obtain the evaluation indexes of the higher education management system.
3.3. Evaluation of Higher Education Management System. The evaluation of higher education management system is an intelligent activity with nonlinear characteristics. In the process of practical work, some evaluation indexes have prominent influence, which is a manifestation of nonlinear characteristics. The prominent impact of indicators is mainly the impact of indicators on the evaluation results, but it cannot be reflected by increasing the weight value. In order to better solve the above problems, it is necessary to establish a fuzzy comprehensive evaluation model, which is a synthetic method based on fuzzy mathematics and gives practical and reliable evaluation results. It converts quantitative evaluation to qualitative evaluation and performs judgement from multiple indexes [21]. The weighted average model is regarded as a special case to fully reflect the outstanding impact of different evaluation indicators. The specific calculation formula is as follows: 


$$
\ddot{y}=\left(a_{1} x_{1}^{a}+a_{2} x_{2}^{a}+\cdots+a_{n} x_{n}^{a}\right)^{1 / e} .
$$

Generally, when all the evaluation indexes of the evaluation objects of the higher education management system are consistent, the evaluation results obtained should also be consistent. However, the evaluation result should be a bounded value. When all the evaluation indexes of the evaluation object are higher than another evaluation object, the evaluation indexes of the former will be significantly better than the latter. This also shows that the maximum increase of each evaluation index will lead to the increase of evaluation index value. At the same time, this increase should be continuous and stable without sudden jump.

The fuzzy evaluation matrix of relative membership degree is obtained through the evaluation index, as shown in formula (6):

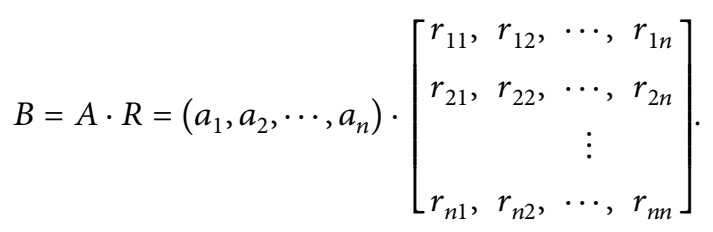

When there are obvious differences in the prominent influence degree of each index, that is, the prominent influence degree of some indexes is small or does not have prominent influence, if some indicators have great outstanding impact and the evaluation values of all indicators are consistent, the evaluation results need to be different from the same indicators.

When evaluating any kind of higher education management system, if there are $m$ evaluation indicators corresponding to factors, the evaluation factor subset can be expressed as

$$
U=\left\{u_{1}, u_{2}, \cdots, u_{m}\right\} .
$$

The evaluator divides the evaluation factors into a set composed of multiple different levels, which is the evaluation set. Assuming that the management mode has $n$ levels, the evaluation level set composed of $n$ levels can be expressed as

$$
V=\left\{v_{1}, v_{2}, \cdots, v_{n}\right\} .
$$

The correlation function is mainly the extent to which the matter-element conforms when the value of the quantity of the matter-element is any point on the real axis [22, 23]. The correlation function is formed by the moments of points and intervals and the modulus of intervals. The specific expression is

$$
K_{i j}=\left\{\begin{array}{ll}
\frac{\rho\left(x_{i}, x_{i j}\right)}{\left[\rho\left(x_{i}, x_{p i}\right)-\rho\left(x_{i}, x_{i j}\right)\right]}, & x \notin x_{i j} \\
-\rho\left(x_{i}, x_{i j}\right), & x_{i} \in x_{i j}
\end{array} .\right.
$$

In the formula, $x_{i j}$ represents the module corresponding to $\left(a_{i j}, b_{i j}\right), \rho$ represents the distance between point and interval, $\rho\left(x_{i}, x_{p i}\right)$ represents the moment of point $x_{i}$ and bounded interval $\left(a_{p i}, b_{p i}\right)$, and $\rho\left(x_{i}, x_{i j}\right)$ represents the moment separation of point $x_{i}$ and bounded interval $\left(a_{i j}, b_{i j}\right)$.

The rating shall be carried out from the first management factor, based on which the correlation degree $T_{i j}$ of the evaluation object to the elements of the evaluation set shall be determined, which mainly represents the correlation degree of the $j$ level of the $i$ management mode. $R$ is set to represent the evaluation result of $i$ management factor, which is called fuzzy evaluation set. The specific expression is as follows:

$$
R=\left\{T_{i j}\right\}=\left[\begin{array}{ccc}
t_{11}, & t_{12}, \cdots, & t_{1 n} \\
t_{21}, & t_{22}, \cdots, & t_{2 n} \\
\vdots \\
t_{n 1}, & t_{n 2}, \cdots, t_{n n}
\end{array}\right] .
$$

The weight reflects the difference between different management modes, and the weight reflects the difference of evaluation marks. The over standard weighting method is used to determine the weight value below. Based on the above, an evaluation model is established to determine the grade. The specific calculation formula is

$$
f(A, X, \Delta)=\left\{\begin{array}{l}
\left(\lambda_{1} x_{1}^{a t}+\lambda_{2} x_{2}^{a t}+\cdots+\lambda_{n} x_{n}^{a t}\right)^{1 / \lambda} \\
\left(\left(\lambda_{1} c_{1}^{a t}+\lambda_{2} c_{2}^{a t}+\cdots+\lambda_{n} c_{n}^{a t}\right)^{1 / \lambda}\right) . \\
{\left[n\left(\lambda_{1} \lambda_{2}\right)^{1 / n}\right]^{1 / \lambda} c^{1 / n \lambda}}
\end{array}\right.
$$

Through the established evaluation model, we can effectively realize the evaluation of higher education management system.

\section{Composition and Optimization of Higher Education Management System}

In order to make full use of the information of higher education management system, reduce communication overhead, maintain the high dynamic load balancing efficiency of intelligent scheduling platform, and improve the parallel efficiency and acceleration ratio of the platform, a parallel scheduling method based on priority principle is designed to facilitate the orderly development of higher education management system.

The platform scheduling model is obtained based on events; that is, there is no dependency between multiuser requests of the information intelligent scheduling platform of higher education management system. Assume that there are $P$ processors in the platform, and the performance architecture of each processor is different. In a certain period of time, there are $L$ independent higher education management system information requests. The operation of the platform is different from the amount of data required for each access request. The processing time required for task $T_{i}$ is $t_{i}$, the amount of data required for higher education management system information access is $H_{1}, H_{2}, \ldots, H_{L}$ in turn, and the highest amount of data is $H_{\max }$. The parallel scheduling 
method created can make effective use of each processor, with roughly the same time and the lowest communication overhead. At this time, only $L>P$ is considered.

For the higher education management information scheduling platform, the traditional scheduling method has the following defects: it cannot give priority to the needs of teaching information access, which improves the waiting time of users. When implementing dynamic scheduling, it cannot ensure that there is the highest amount of teaching management information access request in a period of time. Using the processor with the best performance for task operation also increases the waiting time of users. The processor's processing flow and platform communication cannot be superimposed, which improves the platform communication cost and processor standby time and reduces the platform acceleration ratio.

The scheduling design goal of this method is to maintain the high dynamic load balancing efficiency of the platform, reduce the communication cost and standby time of the platform, and show the priority principle in the elastic strategy. Record the weight of each processor and divide it into three conditions: first, if all processors are idle, the weight $W_{i}$ of the processor is equal to 0 ; second, some processors are idle, the weight of the idle part is 0 , and the processing and weight of nonidle part are the time required to realize the information scheduling request of the current higher education management system; third, all processors are free, and the weight of each processor is the weight given by the platform after the last scheduling termination.

The information scheduling requests in the current period are coded according to the arrival order and arranged in descending order, and the total data $H$ required for all access requests is calculated to obtain

$$
H=L \sum_{i=1}^{L} H_{i}
$$

The processors are arranged in descending order according to their performance, and the sum of the performance of each processor is obtained:

$$
P_{S}=\sum_{i=1}^{P} P_{i}^{1}
$$

In the formula, $P_{i}$ is the performance of the $i$ th processor. The lower the $P_{i}$ value, the better the processor performance.

After the task is assigned for the first time, the maximum number of the remaining access requests is $\left[\begin{array}{l}P \\ 2\end{array}\right]$. Assuming that the number of the remaining access requests is $K$, $K \leq\left[\begin{array}{l}P \\ 2\end{array}\right]$ is guaranteed. The allocation standard of the second higher education management information scheduling access request is to allocate the remaining access requests with the highest amount of data to the processor with the least processing time until the allocation is completed.

Give a new weight to the processor, calculate the specific time, communication time, and idle waiting time for the processor to realize the request under the reassigned access request, and obtain

$$
t_{i}=X_{i} * P_{i}+2 * a+\frac{H_{K i}}{\beta}
$$

The platform load balancing efficiency is

$$
\eta=\frac{t_{S}}{\operatorname{Max}\left\{t_{i}\right\} \times P}
$$

In the formula, $\lambda$ represents a fixed value of the platform. If $\eta<\lambda$, the second task allocation will be implemented again. If all processors have processed the allocated access requests, the platform access request queue is blank, and the method is terminated to complete the composition and optimization of the higher education management system.

\section{Experimental Analysis}

In order to verify the composition and optimization of the higher education management system based on data mining technology, an experiment is designed. It can be seen from the previous content that the higher education management platform built has two processors. $P_{i}$ represents the reading and writing performance of the processor, and the unit is second/megabyte. There are 10 intelligent scheduling requests for higher education management information. Before the simulation, all processors are idle; that is, the weight is 0 . In order to verify the reliability of this method, the simulation scheduling performance comparison experiment is carried out with the methods in reference [3] and reference [4]. The experimental results are shown in Table 1.

Parallel efficiency refers to how a system makes use of parallel processors to enhance performance. Each and every part of a program sometimes may not be parallelized and if this is the case with most of the parts of a program, its performance would be adversely affected [24]. Load balancing efficiency refers to how better a system can distribute tasks to the available resources to enhance the overall performance [25]. A higher acceleration ratio means the system would perform better in case of more cores or computers [26]. From Table 1, we can see that the evaluation indexes of this method are better than those of the two other methods we studied, and the performance and effectiveness of this method are high. This is because the MVC framework of higher education management is used to describe the common logic of the platform by means of strategy planning and callback mode, so as to ensure the loose coupling of the scheduler and maintain the efficient operation of the higher education management system.

The influence of parameters on higher education management system can be divided into two aspects: one is the effect of parameters on load balancing, and the other is the effect of parameters on parallel efficiency and speedup. In the simulation experiment, if the number of processors is small and the performance is different, the dynamic load balancing efficiency and the parallel efficiency will be affected, but the speedup ratio will not change; if the number of processors is large and the performance is low, then the dynamic load balancing efficiency, the parallel efficiency, and the speedup ratio will be very large, as shown in Figure 7. 
TABLE 1: Comparison of scheduling performances of the three methods.

\begin{tabular}{lccccc}
\hline Method & Parallel efficiency & Load balancing efficiency & Acceleration ratio & Parallel time (s) & Total time (s) \\
\hline The proposed method & $89.7 \%$ & $99.6 \%$ & 2.71 & 421.3 & 1241.6 \\
Reference [3] method & $71.2 \%$ & $95.3 \%$ & 1.93 & 574.9 & 1652.4 \\
Reference [4] method & $78.3 \%$ & $95.7 \%$ & 1.95 & 598.1 & 1671.2 \\
\hline
\end{tabular}

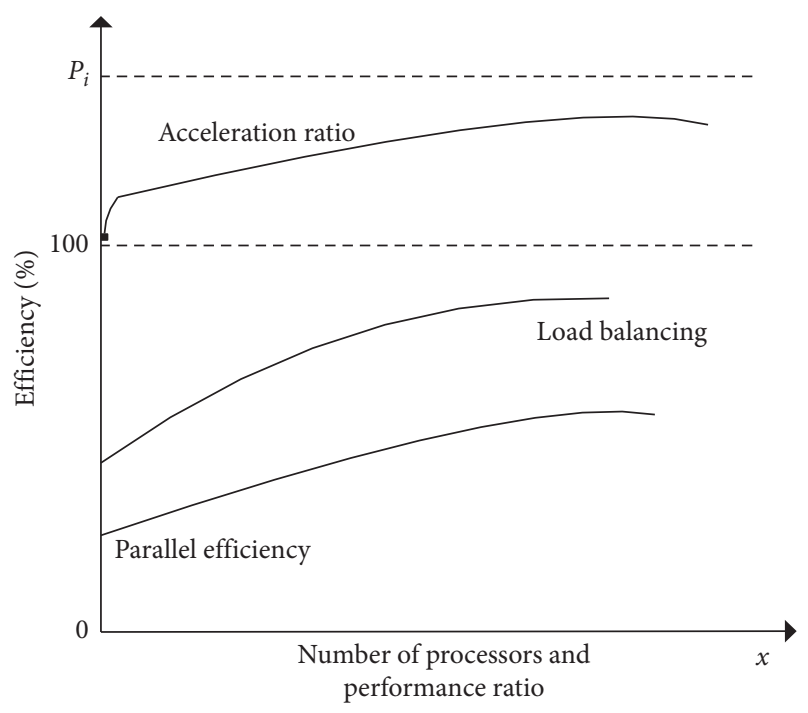

FIgURE 7: Relationship between number of processors and performance.

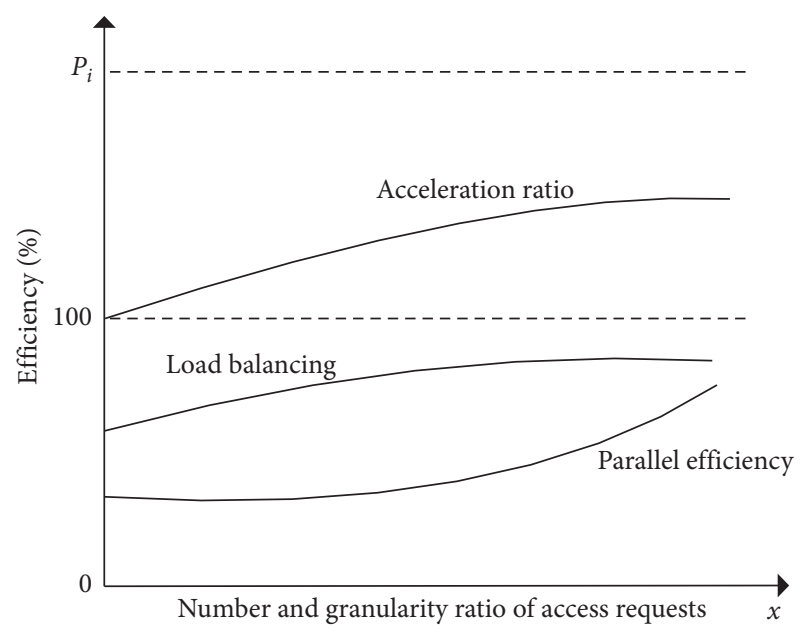

FIGURE 8: Efficiency curves for number of requests and granularity.

In a certain period of time, if the number of requests is much higher than the number of processors, and the granularity of requests is not large, the dynamic load balancing, parallel efficiency, and speedup ratio of parallel scheduling method are very high, as shown in Figure 8.

As can be seen from Figure 8, if the processor is idle for two consecutive periods, the communication cost of the first access request can be ignored. In this case, the dynamic load balancing efficiency, parallel efficiency, and acceleration ratio of the higher education management system built will maintain a high level. This proves that the composition and optimization of higher education management system have good characteristics, effectively deal with the problems of higher education curriculum adjustment and classroom allocation, and provide sufficient conditions for the flexible teaching of higher education management system.

\section{Conclusion}

In this paper, a higher education management system based on data mining technology is constructed to improve the low efficiency of the higher education information systems and tackle the issue of unequal distribution of curriculum resources. We construct the MVC model 2 framework of higher education management platform which is one of the powerful frameworks used for developing huge projects with ease. We collect the data structure in the database, extract the evaluation indicators of higher education management system, separate the display results and business logic, and improve code reusability. We adopt data input, curriculum management, curriculum scheduling, teacher scheduling, and system maintenance and help to form and optimize the higher education management system. The experimental results show that our proposed system can maintain good scheduling performance and provide better teaching services for teachers and students. This method can quickly query the teaching course information of higher education management system, reasonably arrange the teaching details and teacher resources, and provide students with better teaching services. But in the structure and optimization design, in case of the integration of linear programming and numerical analysis, the effect will be better, which is the next step in the research direction.

\section{Data Availability}

The data used to support the findings of this study are available from the corresponding author upon request.

\section{Conflicts of Interest}

The authors declare that there are no conflicts of interest.

\section{Acknowledgments}

This work was supported by the Hainan Higher Education Reform Research Project (no. Hnig2020-123) and 2019 Hainan Basic and Application Basic Research Plan (Natural Science) High-level Talents Project (no. 2019RC247).

\section{References}

[1] V. Kourgiozou, A. Commin, M. Dowson, D. Rovas, and D. Mumovic, "Scalable pathways to net zero carbon in the UK higher education sector: a systematic review of smart energy 
systems in university campuses," Renewable and Sustainable Energy Reviews, vol. 147, no. 2021, Article ID 111234, 2021.

[2] R. O. Vrielink, E. A. Jansen, E. W. Hans, and J. V. Hillegersberg, "Practices in timetabling in higher education institutions: a systematic review," Annals of Operations Research, vol. 275, no. 2, pp. 145-160, 2019.

[3] E. G. Gutsu, N. N. Demeneva, T. V. Mayasova, V. Oksana, and J. Thompson, "Current problem of education management: subjective attitude of university teachers to changes in higher education," Universal Journal of Educational Research, vol. 8, no. 11, pp. 6036-6041, 2020.

[4] A. Z. Djuraevna, "Formation of student management activities in the higher education management system," Psychology and Education Journal, vol. 58, no. 2, pp. 1494-1499, 2021.

[5] Y. Chen, "Optimization of clinical nursing management system based on data mining," Complexity, vol. 2021, Article ID 2110154, 2021.

[6] C. L. Wang, "Research on the core technology of education big data based on data mining," in Proceedings of the 2021 IEEE 6th International Conference on Big Data Analytics (ICBDA), pp. 5-8, IEEE, Guangzhou, China, March 2021.

[7] X. Yin, "Construction of student information management system based on data mining and clustering algorithm," Complexity, vol. 2021, Article ID 4447045, 2021.

[8] F. Aman, A. Rauf, R. Ali, F. Iqbal, and A. M. Khattak, "A predictive model for predicting students academic performance," in Proceedings of the 2019 10th International Conference on Information, Intelligence, Systems and Applications (IISA), pp. 1-4, IEEE, Patras, Greece, July 2019.

[9] L.-J. Y. Shannon, M. Rice, and M. Rice, "Scoring the open source learning management systems," International Journal of Information and Education Technology, vol. 7, no. 6, pp. 432-436, 2017.

[10] C. Scanlan, A. Goyal, and A. A. Spagnolo, "Faculty-driven head-to-head comparison of moodle vs. sakai," in Proceedings of the InE-Learn: World Conference on E-Learning in Corporate, Government, Healthcare, and Higher Education, pp. 891-896, Association for the Advancement of Computing in Education (AACE), Montreal, Canada, October 2011.

[11] H. Tian, J. Zhao, and J. Shen, "Research on optimized storage and analysis system of web log based on django's MVC framework," Journal of Physics: Conference Series, vol. 1769, no. 1, Article ID 012065, 2021.

[12] A. Majeed and I. Rauf, "MVC architecture: a detailed insight to the modern web applications development," Peer Review Journal of Solar \& Photoenergy Systems, vol. 1, no. 1, pp. 1-7, 2018.

[13] R. Liu, J. Liu, Z. Jiang, X. Fan, and Z. Luo, “A bilevel integrated model with data-driven layer ensemble for multi-modality image fusion," IEEE Transactions on Image Processing, vol. 30, no. 12, pp. 1261-1274, 2020.

[14] I. R. Gafurov, M. R. Safiullin, E. M. Akhmetshin, A. R. Gapsalamov, and V. L. Vasilev, "Change of the higher education paradigm in the context of digital transformation: from resource management to access control," International Journal of Higher Education, vol. 9, no. 3, pp. 71-81, 2020.

[15] I. Zhukovskaya, S. Begicheva, and D. Nazarov, "Innovative approach to higher education management as an important factor of sustainable economic development," E3S Web of Conferences, vol. 208, no. 20, Article ID 09018, 2020.

[16] J. Wan, X. Cao, K. Yao, D. Yang, E. Peng, and Y. Cao, "Data mining technology application in false text information recognition," Mobile Information Systems, vol. 2021, no. 4, 13 pages, 2021.
[17] Q. Zheng, Y. Li, and J. Cao, "Application of data mining technology in alarm analysis of communication network," Computer Communications, vol. 163, no. 8, pp. 84-90, 2020.

[18] T. T. Tang, "Supply chain demand forecasting model based on ARIMA and BP neural network and its comparative analysis," Advances in Applied Mathematics, vol. 10, no. 6, pp. 20412049, 2021.

[19] S. Q. Liu and J. Hu, "Double stream network architecture behavior recognition hidden layer model simulation," Computer Simulation, vol. 36, no. 8, pp. 394-398, 2019.

[20] V. Vieira, R. Coelho, and F. M. Assis, "Hilbert-huang-hurstbased non-linear acoustic feature vector for emotion classification with stochastic models and learning systems," IET Signal Processing, vol. 14, no. 8, pp. 522-532, 2020.

[21] P. Zhang and G. Feng, "Application of fuzzy comprehensive evaluation to evaluate the effect of water flooding development," Journal of Petroleum Exploration and Production Technology, vol. 8, no. 4, pp. 1455-1463, 2018.

[22] S. Saga, A. Taruya, M.-A. Breton, and Y. Rasera, "Modelling the asymmetry of the halo cross-correlation function with relativistic effects at quasi-linear scales," Monthly Notices of the Royal Astronomical Society, vol. 498, no. 1, pp. 981-1001, 2020.

[23] D. Bertacca, "Generalization of the kaiser rocket effect in general relativity in the wide-angle galaxy 2-point correlation function," International Journal of Modern Physics D, vol. 29, no. 12, Article ID 2050085, 2020.

[24] D. A. Janies and W. C. Wheeler, "Efficiency of parallel direct optimization," Cladistics, vol. 17, no. 1, pp. S71-S82, 2001.

[25] M. E. Mustafa, "LOAD balancing algorithms round-robin (RR), leastconnection, and least loaded efficiency," Computer Science and Telecommunications, vol. 51, no. 1, 2017.

[26] L. Bu, H. Zhang, H. Xing, and L. Wu, "Research on parallel data processing of data mining platform in the background of cloud computing," Journal of Intelligent Systems, vol. 30, no. 1, pp. 479-486, 2021. 